\title{
Logistic Regression with Tangent Space based Cross-Subject Learning for Enhancing Motor Imagery Classification
}

\author{
Pramod Gaur, Anirban Chowdhury, Karl McCreadie, Ram Bilas Pachori, Senior Member, IEEE, and
} Hui Wang, Senior Member, IEEE,

\begin{abstract}
Brain-computer interface (BCI) performance is often impacted due to the inherent non-stationarity in the recorded EEG signals coupled with a high variability across subjects. This study proposes a novel method using Logistic Regression with Tangent Space-based Transfer Learning (LR-TSTL) for motor imagery (MI)-based BCI classification problems. The singletrial covariance matrix $(\mathrm{CM})$ features computed from the EEG signals are transformed into a Riemannian geometry frame and tangent space features are computed by considering the lower triangular matrix. These are then further classified using the logistic regression model to improve classification accuracy. The performance of LR-TSTL is tested on healthy subjects' dataset as well as on stroke patients' dataset. As compared to existing within-subject learning approaches the proposed method gave an equivalent or better performance in terms of average classification accuracy $(\mathbf{7 8 . 9 5} \pm \mathbf{1 1 . 6 8 \%})$, while applied as leaveone-out cross-subject learning for healthy subjects. Interestingly, for the patient dataset LR-TSTL significantly $(p<0.05)$ outperformed the current benchmark performance by achieving an average classification accuracy of $81.75 \pm 6.88 \%$. The results show that the proposed method for cross-subject learning has the potential to realize the next generation of calibration-free BCI technologies with enhanced practical usability especially in the case of neurorehabilitative BCI designs for stroke patients.
\end{abstract}

Index Terms-Brain-computer interface, EEG, motor-imagery, logistic regression, Riemannian geometry, tangent space, transfer learning.

\section{INTRODUCTION}

A brain-computer interface $(\mathrm{BCI})$ provides a means of direct communication for controlling external assistive machines and can be used by motor-impaired people with damaged neuromuscular pathways [1] by converting neuro-physiological signals into control commands [2]. The EEG based brain connectivity has been used for the diagonosis of post-traumatic stress disorder (PTSD) [3], [4]. BCI can be implemented by recording electroencephalogram (EEG) data of imagined movement or motor imagery (MI), which is a process of mental imagination by which an individual voluntarily imagines,

Manuscript received XXXX XX, 2020; revised XXXX XX, 2020.

(Corresponding author: Pramod Gaur.)

P. Gaur is with the Department of Computer Science, Birla Institute of Technology \& Science, Pilani - Dubai Campus, Dubai, UAE (e-mail pramodgaur.iitkgp@gmail.com). A. Chowdhury is with School of Computer Science and Electronic Engineering, University of Essex, Colchester, CO4 3SQ, UK (e-mail: a.chowdhury@ essex.ac.uk). K. McCreadie, and Hui Wang are with the Ulster University, UK (e-mail: k.mccreadie@ulster.ac.uk, h.wang@ulster.ac.uk). R. B. Pachori is with the Department of Electrical Engineering, IIT Indore, Indore, India (pachori@iiti.ac.in). without actually performing, movement of a body part, e.g. arm, foot, tongue.

EEG-based BCI is easy to perform and does not require invasive surgery, but cannot make use of higher-frequency signals as the skull dampens electromagnetic waves generated from neuronal activity. Although, brainwaves of interest are produced across a range of well-studied frequency bands, these often vary between subjects. Hence, this study attempts to find the optimum frequency ranges for individual subjects to improve classification performance of MI tasks. There are many factors in recorded EEG signals which can affect the BCI performance, such as highl non-stationary, high inter/intra-subject variability [5], and poor spatial resolution. As the motor learning process is different in different people which influences the cortio-subcortical networks to produce variation in the EEG patterns across subjects [6]. The intra-subject variations in the EEG pattens can be caused due to change in different affective changes such as mood, motivation, and mental fatigue which may vary across session or even within a session [7]. In this study, inter-subject or cross-subject transfer learning is used to compute results which are better than those computed using training data from the same subject (withinsubject learning) [8], [9], [10]. Transfer learning is a machine learning method of applying the knowledge learned from one task to a different task but on a similar type of activity [11], [12].

Robinson et al. [13] used a wavelet-based common spatial pattern (CSP) technique employing a Fisher linear discriminantclassifier. They used low-frequency features for the classification of hand movements of various speeds whilstYang et al. [14] classified left and right-hand movements using a time-frequency optimization and linear discriminant analysis (LDA). The CSP algorithm is one popular method used for extracting features which learns spatial filters by minimising the variance of one class while maximising the variance of another [15], [16], [17]. In BCI competitions [18], [19] CSP is one of the most efficient and popular algorithms. To obtain effective discrimination, CSP analysis is applied to band-pass filtered EEG signals [20] and then the co-variance matrix is obtained [21], [22].

However, the CSP algorithm can be improved by using subject-specific parameters. For example, the time period selection can be done optimally rather than heuristically using temporally constrained sparse group spatial pattern (TSGSP) [23]. The inter-subject performance of CSP is also 
improved by using novel sparse group representation model (SGRM) [24]. Blankertz [25] used subject-specific bandpass frequencies to enhance classification accuracy whilst a Filter Bank Common Spatial Pattern (FBCSP) was used to improve CSP performance by Ang [26]. Another method improves CSP by using prior information added to the CSP learning process, in the form of regularization terms, known as Regularized CSP (RCSP) [27], [28], [29], [30]. There has also been an attempt by Lotte et al. to unify these various RCSP algorithms by developing a simple theoretical framework [10].

Recently, filtering methods based on single-/multi-channel empirical mode decomposition (EMD) were studied using statistical measure, namely, the mean/median frequency. These measures were calculated for intrinsic mode functions (IMFs) to remove the artefacts and noise resulting in improved classification of EEG signals [8], [31], [32], [33]. These may be employed in online BCI for artefact removal and denoising in a real-time environment. EMD was first proposed in 1998 by Huang [34]. It decomposes the original signal into IMFs which are a group of finite band-limited basis functions. Similarly, Multivariate empirical mode decomposition (MEMD) has also been proposed utilizing the information from across the channels [8],[35].

In this work, a novel technique is presented by fusing logistic regression with tangent space-based transfer learning (LR-TSTL) for binary classification of EEG data. Additionally, the optimum low and high-frequency ranges for each subject for left vs right hand MI classification has been identified because of the inter-subject variability in contrast to the other studies where a fixed range of $8-30 \mathrm{~Hz}$ is considered [10]. The main contributions of the paper are as follows:

- The novel LR-TSTL method can enhance the crosssubject transfer learning performance to a level comparable to that found when performing the within-subject learning in terms of average classification accuracy when compared to three state-of-the-art within-subject learning methods for healthy subjects.

- In the case stroke patients, the cross-subject transfer learning performance of LR-TSTL significantly outperforms the benchmark within-subject learning performance on the same dataset.

- The proposed method also gives comparable cross-subject learning performance between healthy and stroke patients' data with reduced variability and higher classification accuracy in the case of patients making it a better alternative to realize calibration free setup and allowing for a more usable neurorehabilitative BCI systems.

The remainder of the paper is organized as follows: Section II discusses the datasets for healthy and patients; Section III discusses methods used for BCI; Section IV discusses the proposed methodology: logistic regression with tangent space based transfer learning; Section V presents the results obtained and a comparison with the state-of-the-art results reported in the literature using the same datasets [36], whilst Section VI concludes the study.

\section{DATASET}

The results were obtained on two datasets: one is for healthy subjects and another for stroke patients. For healthy subjects the popular publicly available dataset $2 \mathrm{a}$ from BCI Competition IV was used whilst the dataset generated in the work by Chowdhury et al. [37] was used for patients. The descriptions of these two datasets are given as follows.

\section{A. Healthy subjects' dataset}

Publicly available dataset $2 \mathrm{a}$ from BCI Competition IV has been used for validating the performance of the proposed method on healthy subjects. It includes signals from twentyfive channels, including three left mastoid EOG channels and twenty-two EEG channels. The channel locations are shown in Fig. 2 a). In this dataset, four MI tasks were carried out which includes foot, tongue movement, and two hand tasks, namely, right hand, left hand. The dataset includes nine healthy subjects with two sessions per subject: one for training, and one for testing. For each MI task, there are 72 trials, so each session includes $288 \mathrm{MI}$ data trials. Table $\mathrm{I}$ lists the rejected trials from all subjects:

TABLE I

REJECTED TRIALS FROM BCI COMPETITION IV DATASET 2A FOR NINE SUBJECTS.

\begin{tabular}{c|ccc|cccc}
\hline \multirow{2}{*}{ Subject } & \multicolumn{7}{|c}{ Number of Trials } \\
& Total & CT & RT & LH & RH & Foot & Tongue \\
\hline A01 & 288 & 281 & 7 & 1 & 2 & 3 & 1 \\
A02 & 288 & 283 & 5 & 1 & 1 & 3 & 0 \\
A03 & 288 & 273 & 15 & 5 & 2 & 4 & 4 \\
A04 & 288 & 228 & 60 & 13 & 15 & 13 & 19 \\
A05 & 288 & 276 & 12 & 2 & 7 & 0 & 3 \\
A06 & 288 & 215 & 73 & 19 & 17 & 18 & 19 \\
A07 & 288 & 277 & 11 & 1 & 3 & 1 & 6 \\
A08 & 288 & 271 & 17 & 6 & 4 & 3 & 4 \\
A09 & 288 & 264 & 24 & 7 & 7 & 3 & 7 \\
\hline
\end{tabular}

A recording of around 5 minutes was done at the start of each session to estimate the effect of the EOG. The recording was split into three blocks; 1) two minutes with open eyes (viewing an arrow on-screen), 2) one minute with eyes closed, 3 ) one minute with eye movement. At $t=0 \mathrm{~s}$ a fixation cross is shown on a black screen followed by a brief acoustic warning sound at the start of the experiment. At $t=2 \mathrm{~s}$ an arrow is shown for $1.25 \mathrm{~s}$ and the left, right, down or up direction refers to one of the four groups. The participants were told to execute the MI task till $\mathrm{t}=6 \mathrm{~s}$ after which they have been allowed a short break. The timing diagram of a single trial of the data acquisition paradigm is shown in Fig. 2(c). All band-passed signals were then filtered from 0.5 to $100 \mathrm{~Hz}$. In addition, a 50 $\mathrm{Hz}$ notch filter was added for line noise reduction. The EEG signals reported were sampled at a frequency of $250 \mathrm{~Hz}$. It is to be noted that only left and right MI data was used from this dataset for extracting the binary classification results using the proposed LR-TSTL method.

\section{B. Patients' dataset}

The patient dataset used in this study is from Chowdhury et al. [37], which is composed of 10 hemiparetic chronic 
stroke patients. The EEG channel locations and the timing diagram of the data acquisition protocol are shown in Fig. 2 (b) and Fig. 2 (d) respectively. The motor imagery EEG signals from 10 chronic hemiparetic stroke patients were recorded in two different phases. The data from the first phase was used to train the classifier while no neurofeedback was provided during this phase. The neurofeedback in terms of visual and proprioceptive feedback with a hand exoskeleton was provided during the second phase which was the online BCI phase. The neurofeedback was contingent to the classifier output. There were two runs of 40 trials each during the first phase where the duration of each run was roughly $7 \mathrm{~min}$ and $30 \mathrm{~s}$. This is because each trial consists of $8 \mathrm{~s}$ period with an inter-trialinterval (ITI) of 2-3 s. In each run there were 20 trials for instructing the patients to do left hand imagery and 20 trials to instruct the patients to do right hand imagery which were presented at random to the participants. There was a gap of 16 min between the training and testing (online BCI) phases within which the classifier was trained. The online feedback phase has only one run of 40 trials equally distributed between left and right hand MI. It is to be noted that during the original BCI a SVM classifer was trained using the CSP features on $\mathrm{mu}(8-12 \mathrm{~Hz})$ and beta $(16-24 \mathrm{~Hz})$ bands which were updated during the online BCI phase using a covariate-shift adaptation technique [9]. However, in the current study the proposed LRTSTL method in offline mode was used.

\section{METHODS}

In MI BCI, one of the main tasks is to classify raw brain signals into different classes. However, EEG signals are extremely subject-specific, noise sensitive, and are inherently non-stationary due to changes in signal characteristics not only in frequency but also over time. Subject specific timefrequency selection methods have been proposed in the past to deal with this problem such as using Fisher discriminant analysis-type F-score criterion [38], recursive band elimination [39], and channel-frequency map [40]. This study uses a similar type of subject specific frequency ranges selection for all MI tasks by building on an Multivariate Empirical Mode Decomposition filtering (MEMDF) technique developed in a previous work [33] which is further coupled with the fusion of logistic regression with tangent space based transfer learning in the Riemannian geometry framework. A block diagram of the technique proposed is shown in Fig. 1

\section{A. Multivariate Empirical Mode Decomposition (MEMD)}

The single channel Empirical Mode Decomposition (EMD) method [34] is a data-driven approach for decomposing a signal into a group of finite band-limited basis functions known as intrinsic mode functions (IMFs). Recently, a multivariate analytical mode of decomposition (MEMD) [41] was introduced where, rather than calculating the local mean using an average of both lower and upper envelopes such as traditional EMD, created instead multiple $\mathrm{n}$ dimensional envelopes by projecting the signal in $\mathrm{n}$ variable spaces using MEMD in any path. To achieve the local estimate, such estimates are therefore summed.
Let $\mathrm{n}$ dimensional vectors $e(t)_{t=1}^{T}=e_{1}(t), e_{2}(t) \ldots e_{t}(t)$ denote a multivariate signal where $d_{k}^{\eta}=d_{1}^{k}, d_{2}^{k} \ldots d_{n}^{k}$ denotes a series of direction vectors along the directions provided by angles $\eta^{k}=\eta_{1}^{k}, \eta_{2}^{k} \ldots \eta_{n-1}^{k}$ on a $(n-1)$ sphere. Calculation measures for MEMD are shown below:

- Choose appropriate points on a $(n-1)$ sphere to examine.

- Calculate the projection $p^{\eta_{k}(t)_{t}=1^{T}}$ of the input signal into the path vector for all $\mathrm{k}$ resulting $p^{\eta_{k}(t)_{k}=1^{K}}$ as the projection collection.

- Find the instants in time $t_{i}^{\eta_{k}}$ that lead to the maxima in $p^{\eta_{k}(t)} \underset{k=1}{K}$.

- Interpolate $t_{i}^{\eta_{k}}, e\left(t_{i}^{\eta_{k}}\right)$ to receive curves $c^{\eta_{k}(t)}{ }_{k=1}^{K}$ in multivariate envelopes.

- Calculate the mean mean $(t)$ of $\mathrm{K}$ path vector envelopes

$$
\operatorname{mean}(t)=\frac{1}{k} \sum_{k=1}^{K} c_{k}^{\eta}(t)
$$

- Subtract the local mean from direction vector $L(t)=$ $d(t)-\operatorname{mean}(t)$ for extracting details. If with $L(t)$ convergence IMF requirement is satisfied, apply the method to $d(t)-$ mean $(t)$, otherwise repeat until $L(t)$ becomes IMF.

All Multivariate Intrinsic Mode Functions (MIMFs) will follow two criteria: 1) the mean value of the envelope should be nil at every point, dictated by the local minima and the local maxima, 2) the number of minima and maxima should be nil or vary by just one. For a detailed explanation please see [41]. All twenty-two EEG channels from the dataset are used in this work (Fig. 2(a)). A single trial of foot MI from subject A09T is used to decompose the EEG signal from channels $\mathrm{FC} 2, \mathrm{C} 2$, and $\mathrm{CP} 2$ to gain a deeper insight into the MEMD decomposition which is displayed in Fig. 3. All MI EEG signals were then decomposed into tongue, feet, left, and right hand signals utilizing the MEMD system.

A MEMD filtering helped to identified variable bands around the mu and beta bands (8-30) $\mathrm{Hz}$ for each subject to filter training and testing EEG signals for all motor imagery tasks in the preprocessing step. This filtering method helps screen the subject specific $m u$ and beta bands by keeping the IMFs that belong to them whilst discarding all noise and artefacts as EEG signals are highly subject dependent. Discarded IMFs include components of high $(>30 \mathrm{~Hz})$ and low $(<8 \mathrm{~Hz})$ frequency which may include artefacts and noise. The filtering criteria used to select the IMFs utilizes the mean frequency of these obtained IMFs and then retaining IMFs whose mean frequency falls in the range of $8-30 \mathrm{~Hz}$. The mathematical formula of the mean frequency(MFQ) is expressed [12], [42] as:

$$
M F Q_{I M F}=\frac{\sum_{a=1}^{m} P_{a} f b_{a}}{\sum_{a=1}^{n} P_{a}}
$$

Where $m$ denotes the frequency bin length and $P_{a}$ provides the frequency bin range $a$, while $f b_{a}$ represents the frequency bin $a$. The IMFs obtained that fulfil the above requirements are then summed to get this enhanced EEG signal. This research study presents a fusion of Subject specific MEMD (SSMEMD) and logistic regression with tangent space features in 


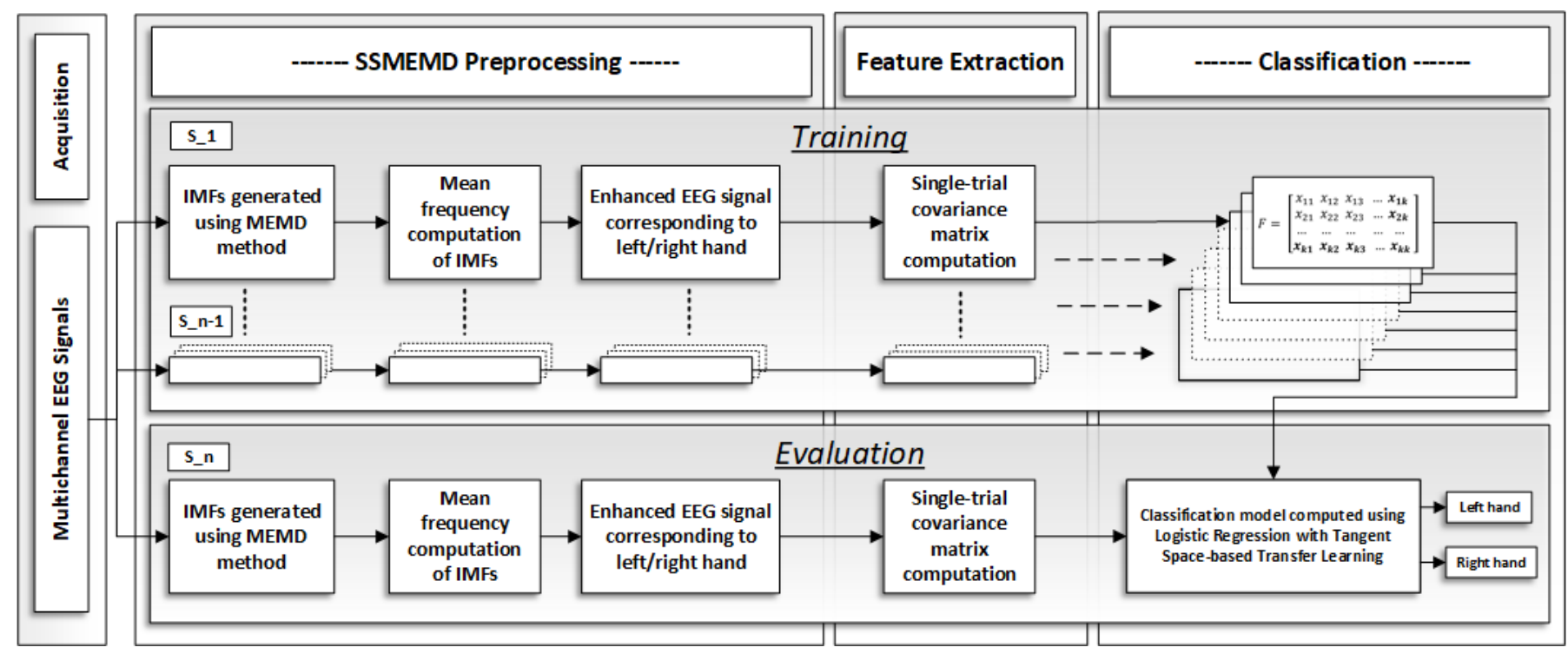

Fig. 1. Overview of the proposed work showing Subject-Specific Multivariate Empirical Mode Decomposition (SSMEMD) preprocessing stage and logistic regression based transfer learning computation.

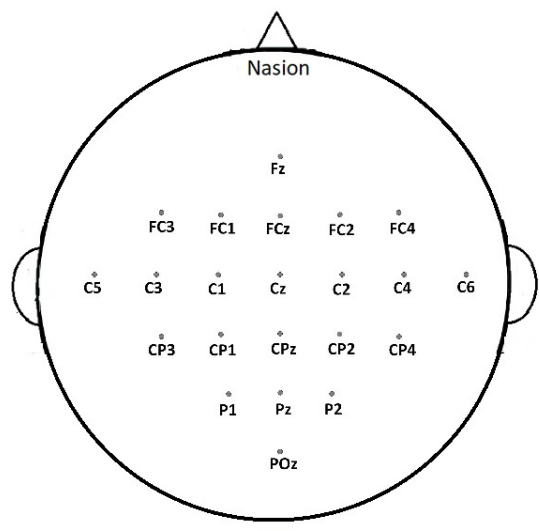

(a)

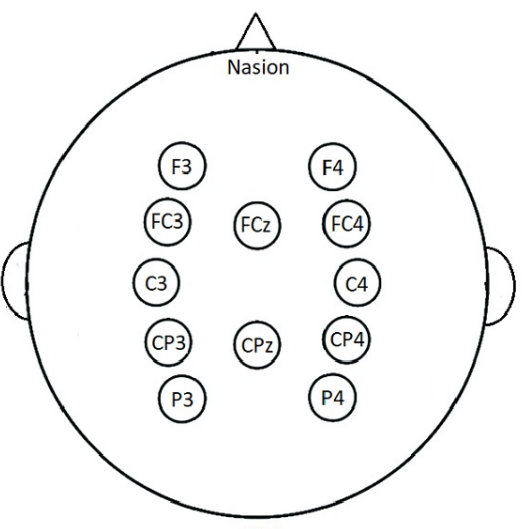

(b)

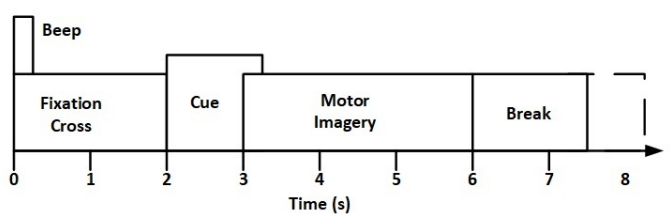

(c)

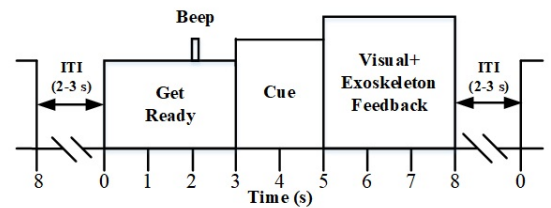

(d)

Fig. 2. EEG channel locations and timing diagram of the data acquisition from the two different datasets used in the study. (a) 22 Channel locations of BCI Competition dataset IV-2a (Healthy); (b) 12 Channel locations of the patients' dataset; (c) Timing diagram of BCI Competition dataset IV-2a (Healthy); (d) Timing diagram of the patient dataset.

the Riemannian geometry framework for two-class classification. Using this EEG framework to minimise dimensionality, produces a bilinear logistic regression model that can learn specific selected features from two MI tasks.

\section{Proposed Methodology : Logistic Regression With TANGent SPACE BASED TransFer LEARning}

\section{(LR-TSTL)}

One issue with BCI is that every subject's EEG pattern is different and is highly non-stationarity resulting in inter/intra-subject non-stationarity. Also, long training sessions are often needed before an testing/evaluation session is possible. Previous research aimed to resolve this problem by introducing a novel subject-independent classification model that was used without the need for training session data [12].
In transfer learning, experience from a previously learned solution can be applied to train a new model, whilst also addressing the issue of lack of data. When analysing and classifying EEG signals, the direct estimation and manipulation of EEG signal co-variance matrices can be obtained for source extraction. The co-variance method, also known as the Riemannian technique [43], encapsulates signals dependent on energy knowledge of EEG signals. The raw data is expressed as an input to the spatial co-variance matrix. It is often assumed that spatial co-variance matrices are on the highdimensional positive definite matrices (SPD) manifolds [44]. A real matrix of dimension $n \times n$ is called as symmetrix positivedefinite (SPD) if and only if the scalar $z^{T} M z$ is strictly positive for all non-zero column vector $z$ of $n$ real numbers. A SPD matrix is always diagonalizable with strictly real positive eigenvalues. The manifold topology of these SPD matrices 

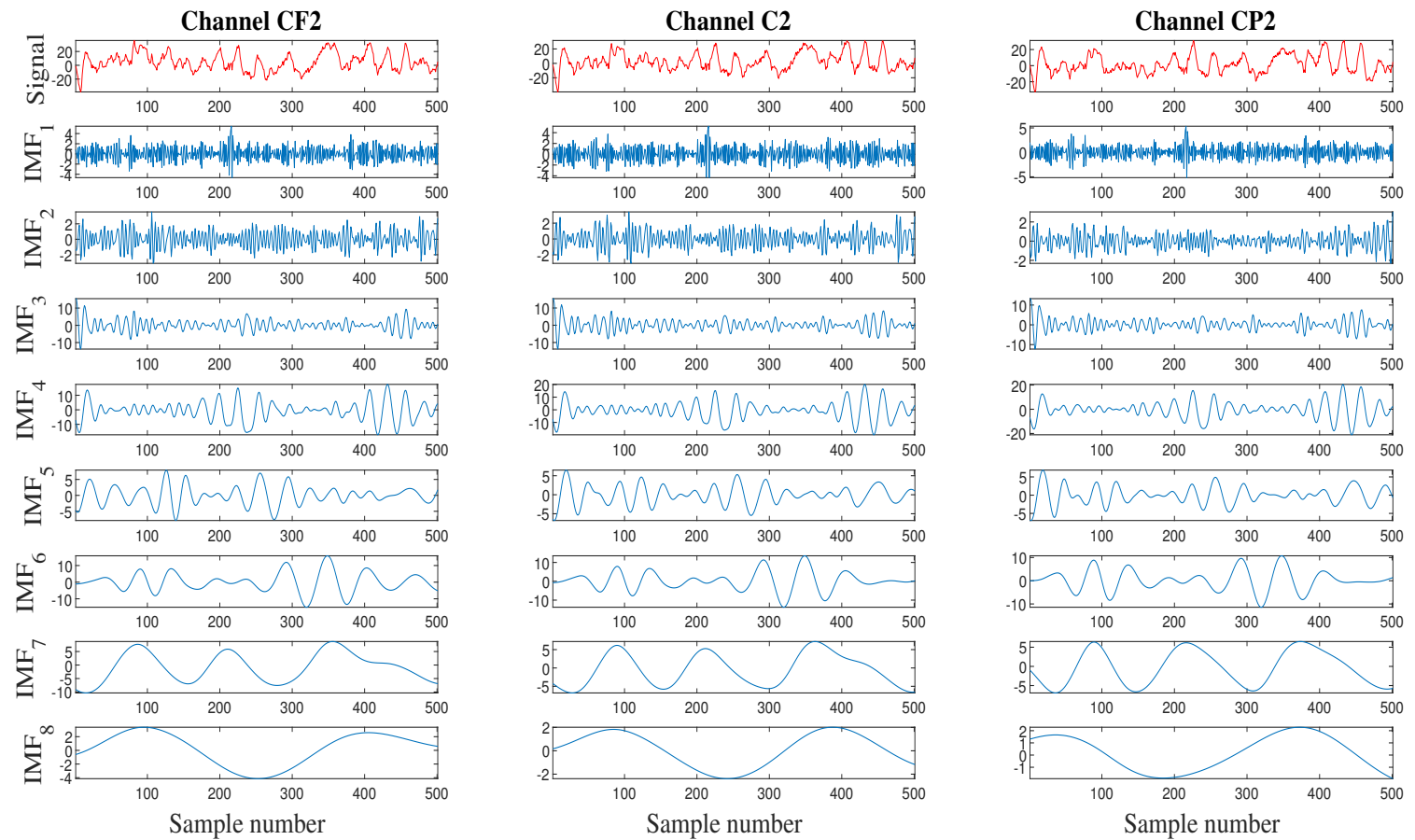

Fig. 3. Simultaneous decomposition of three EEG channels signal FC2, C2 and CP2 and their corresponding MIMFs from Subject A09T for foot MI task.

are based on Riemannian Geometry (RG) and can be used for classification purpose. An interesting property of the SPD manifolds is that it enables the use of explicit formulae for easier implementation. In this regard it is important to note that the space of SPD matrices can be differentiable in a Riemannian Manifold [45] and also the space of SPD is invariant by projection. This SPD matrix is a linear map with undetermined weights from the high-dimensional SPD manifold to a low-dimensional one. Next, is to project the matrices to its tangent space on SPD manifolds, which is a local linear approximation of curved space. The space of the SPD defined by a differentiable Riemannian Manifold $(\mathcal{M})$ can be denoted as $V(r)$ where the tangent space for the derivatives of a matrix $V$ lies in a vector space over $\mathcal{M}$. Now if we take the derivative of the geodesic distance between exponential mapping $V_{i}=\operatorname{Exp}_{V}\left(U_{i}\right)$ and $V$ at $t=0$, this would the tangent vectors $U_{i}$ defined as,

$$
\operatorname{Exp}_{V}\left(U_{i}\right)=V_{i}=V^{1 / 2} \exp \left(V^{-1 / 2} U_{i} V^{-1 / 2}\right) V^{1 / 2}
$$

Here the inverse mapping is obtained by the logarithmic mapping defined as,

$$
\log _{V}\left(V_{i}\right)=U_{i}=\operatorname{lower}\left\{V^{1 / 2} \log \left(V^{-1 / 2} V_{i} V^{-1 / 2}\right) V^{1 / 2}\right\}
$$

More details about the geometric procedure can be found in [43] and [8].

Only $\mathrm{k}(\mathrm{k}+1) / 2$ is taken into account by considering the lower or higher triangular matrix of the $\mathrm{k} \mathrm{x} \mathrm{k}$ features given because of the symmetric nature of the matrix. Since the sample co-variance matrices (SCM) are symmetric, for this analysis the lower triangular matrix is called giving a total of $d=k(k+1) / 2$ attributes. These features, which are used for the classification as inputs or independent variables to the logistic regression model, are determined from tangent space. A logistic regression classifier with tangent space feature in the Riemannian geometry framework is used in this paper to define the four groups of MI EEG features obtained from the tangent space. This logistic regression model is built with seven independent variables to estimate the outcome of the four-class MI activities known as the dependent variable.

For a dataset where $z_{1}, z_{2}, z_{3} \ldots, z_{k}$ there is an input matrix and $\mathrm{y}$ is its class labels. $z_{1}, z_{2}, \ldots z_{k}$ are regarded as independent variables / predictors and $\mathrm{y}$ is the dependent variable. In the context of logistic regression, the probability of the dependent variable $y$, if $y$ resides in class I, can be defined: [46]

$$
P\left(y=1 \mid z_{1} \ldots z_{k}\right)=\pi=\frac{e^{\mu_{0}+\sum_{k=1}^{\infty} \mu_{i} z_{i}}}{1+e^{\mu_{0}+\sum_{k=1}^{\infty} \mu_{i} z_{i}}}
$$

Where, $\pi$ is a conditional probability in the form $P(y=$ $\left.1 \mid z_{1}, \ldots, z_{k}\right)$, the probability of $\mathrm{y}$, when $\mathrm{y}$ resides in class 0 , is computed as $1-\pi=1-P\left(y=1 \mid z_{1}, \ldots, z_{k}\right)=$ $P\left(y=0 \mid z_{1}, z_{2}, \ldots, z_{k}\right)$. In the above equation, $\beta_{0}$ is an intercept and $\beta_{1}, \beta_{2}, \ldots, \beta_{k}$ are the regression coefficient related to independent variables $z_{1}, z_{2}, z_{3}, \ldots, z_{k}$. Similarly, we can calculate this for other remaining classes. Such parameters are calculated using the technique of maximum probability estimation (MPE). Note that a linear relationship between the dependent and independent variables is not commonly 
assumed in logistic regression and it does not include independent variables which are normally distributed. Here MI can be considered the dependent variable, called $\mathrm{y}$, and the seven statistical characteristics can be viewed as seven independent variables $(\mathrm{k}=7)$.

\section{RESULTS AND DISCUSSION}

There are four MI tasks in the BCI Competition IV-2a dataset with six combinations of binary MI tasks as follows: right hand versus foot $(\mathrm{RvF})$, right hand versus tongue (RvT), left hand versus right hand (LvR), left hand versus foot (LvF), left hand versus tongue (LvT), and foot versus tongue (FvT). Among these binary class combinations, only results for the LvR combination are given. This is because the majority of BCI paradigms are based on this approach and hence comparison of the results with other existing studies can easily be made. Unlike the conventional approach of performing within subject classification this study focuses on the crosssubject approach as it is one of the popular ways to realize a calibration free BCI for a robust and reliable practical implementation of this technology.

The cross-subject test accuracies for BCI Competition IV-2a dataset (LvR) are given in Table $\Pi$ along with the individualized low and high frequency ranges for feature extraction. The cross-subject accuracies are computed following the leaveone-out approach whereby the accuracy is calculated for a particular subject using the training data from the rest of subjects to build the classifier leaving out the particular test subject. The low and high frequency range has been identified for each subject with a mix of automatic and manual inspection and further, this identified low and high frequency range was used in the evaluation process to account for the inter-subject variability in the frequency response. It is to be noted that for all comparisons between results we have used Wilcoxon signed rank test with a $p$-value threshold of 0.05 as this is a non-parametric test and the comparison were made within the same population.

Table II shows that the average cross-subject accuracy across 9 subjects in BCI Competition Dataset IV-2a in LvR case is $78.95 \%$ ( \pm 11.68 ) by applying the proposed LRTSTL method. The highest classification accuracy (CA) is noted in subject $\mathrm{A} 08(\mathrm{CA}=94.78 \%)$ and the lowest $\mathrm{CA}$ is noted in subject $\mathrm{A} 02(\mathrm{CA}=63.38 \%)$. A comparison of the classification performance of the proposed method with other state-of-the-art methods (M1 [8], M2 [9], and M3 [10]) is given in Table II Method 1 (M1) contains results obtained from a study by Gaur [8], which uses a subject-specific multivariate empirical mode decomposition based filtering method (SS-MEMDBF) for preprocessing and uses a Riemannian geometry framework, made separately for each subject for binary classification. The mean classification accuracy (MCA) of the proposed method $(78.95 \% \pm 11.38)$ is comparable to the method M1 at $79.93 \%( \pm 14.99)$ as there was no significant difference in performance $(p>0.05)$. Notably, 3 out of the 9 subjects have shown improvement, while the variability in performance was reduced as revealed by a decrement in standard deviation (Std) of the accuracy of all subjects by
3.31\%. Method 2 (M2) uses CSP and uses covariate shift detection along with adaptive learning [9]. The MCA of the proposed method $(78.95 \% \pm 11.68)$ is higher on comparison with method M2 (74.92\% \pm 15.43$)$. Notably, 6 out of 9 subjects show improvement with an overall improvement of $+4.03 \%$ in MCA with a reduction in standard deviation of $3.75 \%$. Method 3 (M3) uses band-pass filtered EEG data between 8 and 30 $\mathrm{Hz}$ to compute CSP and then calculates the log variance taken from three pairs of filters for extraction of features coupled with LDA for a two class classification problem [10]. The MCA of the proposed method $(78.95 \% \pm 11.68)$ is higher when compared with method M3 $(78.01 \% \pm 17.01)$ by $+0.94 \%$ with a reduced standard deviation of $5.33 \%$. Notably, 6 out of the 9 subjects have shown improvement.

However, it is to be noted that the M1, M2, and M3 are all applied following the within-subject approach where it is comparatively easy to obtain higher accuracy as the classifier is trained on the same subject's training data. On the contrary, it is comparatively difficult to get adequate performance using a cross-subject approach as a mix of training data from several other subjects' are used in this case which generally have a high degree of inter-subject variability. Hence, unless the proposed method is able reveal more generalizable features across all the different subjects the classifier is prone to poorer performance. This is also evident from the fact that as compared to all the other methods (M1, M2, and M3), the proposed method got $3.31 \%$ to $5.33 \%$ lower standard deviation against the average classification accuracy across all the subjects. Interestingly, we can see that the proposed method has outperformed the M2 method (with $4.03 \%$ higher average accuracy) which not only used the state-of-the-art common spatial filtering (CSP) feature but also used covariate shift detection based adaptive classification. In our case, this improvement is achieved without adaptation (as the classifier was not updated during testing) and more importantly following the cross-subject approach. The proposed method also achieved a slightly higher average accuracy (+0.94\%) than M3 which used regularised-CSP features in a withinsubject approach. Although the M1 method, which used a similar MEMD features in within-subject approach, achieved a slightly higher performance $(+0.98 \%)$ than the proposed method, the difference is not significant $(p<0.05)$. Thus, the proposed method has given comparable or better performance in cross-subject classification than the methods used in withinsubject classification.

Such promising results encouraged further exploration and hence LR-TSTL was applied on a more challenging dataset involving stroke patients. It is well-known that due to the presence of altered neuro-dynamics after stroke data nonstationarity and inter-subject variability effects are higher in stroke patients' EEG data than in healthy subjects [7]. Hence, a pertinent question is whether the proposed method would be able to maintain its superiority not only on a healthy subject dataset (as already shown in the case of BCI competition IV2a) but also on patient data. For this purpose, the performance of LR-TSTL is shown on a previously used hemiparetic patients' dataset [37], where the patients performed left and right hand motor task while wearing a hand-exoskeleton on 
TABLE II

LOW AND HIGH-FREQUENCY VALUES IN HZ WITH CROSS-SUBJECT CLASSIFICATION ACCURACY (\%) FOR THE PROPOSED METHOD (LR-TSTL) ON LEFT VERSUS RIGHT HAND TASK.

\begin{tabular}{ccccccc}
\hline Subject & $\begin{array}{c}\text { Low } \\
\text { Frequency }\end{array}$ & $\begin{array}{c}\text { High } \\
\text { Frequency }\end{array}$ & $\begin{array}{c}\text { Proposed } \\
\text { Method LvR }\end{array}$ & M1 & M2 & M3 \\
\hline A01 & 9 & 27 & 89.36 & 91.49 & 90.28 & 88.89 \\
A02 & 7 & 26 & 63.38 & 60.56 & 57.64 & 51.39 \\
A03 & 6 & 24 & 92.7 & 94.16 & 95.14 & 96.53 \\
A04 & 9 & 24 & 76.72 & 76.72 & 65.97 & 70.14 \\
A05 & 9 & 28 & 63.7 & 58.52 & 61.11 & 54.86 \\
A06 & 8 & 26 & 74.07 & 68.52 & 65.28 & 71.53 \\
A07 & 10 & 29 & 73.57 & 78.57 & 61.11 & 81.25 \\
A08 & 7 & 26 & 94.78 & 97.01 & 91.67 & 93.75 \\
A09 & 9 & 24 & 82.31 & 93.85 & 86.11 & 93.75 \\
\hline Average & & & 78.95 & 79.93 & 74.92 & 78.01 \\
Std & & & 11.68 & 79.93 & 74.92 & 78.01 \\
p-value & & & & 0.89 & 0.07 & 0.62 \\
\hline
\end{tabular}

their impaired hand. The classification accuracy along with kappa values, and the low and the high frequency ranges for feature extraction are shown in Table III] The average classification accuracy across all the patients is found to be $81.75 \%$ ( \pm 6.88$)$, while the average kappa value is 0.635 $( \pm 0.13)$. The highest accuracy can be noted for patient P08 (92.5\%), while the lowest accuracy can be noted for P06 (75\%). The right most three columns in Table III shows the comparison with previously published results on the same patient dataset. The EEG-NAC and EEG-CSAC methods are CSP based methods with a difference that the former is nonadaptive and the later is adaptive [37]. The last column shows the results with EEGNet which is a convolutional neural network based method [47] applied on the same dataset published recently [48]. Using EEG-NAC the accuracy on this dataset was $70.25 \%$ (Kappa $=0.41)$ using CSP features with SVM classifier in non-adaptive mode (EEG-NAC) [37], while in adaptive mode using covariate-shift adaption (EEGCSAC) the average accuracy was $75.75 \%$ (Kappa $=0.52$ ). In comparison to the benchmark the proposed LR-TSTL significantly outperformed $(p<0.05)$ the EEG-NAC both in terms of classification accuracy and kappa value. The proposed method also performed better than EEG-CSAC as there is an average improvement in the accuracy $(+6 \%)$ and kappa value $(+0.12)$, although the $p$-value $(p=0.0625)$ failed to achive the statistical significance threshold of 0.05 by a small margin which can be primarily attributed to the small sample size. Moreover, it is important to note that this improvement in performance over EEG-CSAC is achieved without any adaptation during the decoding of test data while EEG-CSAC updated the classifier with newly available data on every covariate shift detection. Another important fact to be considered is that LR-TSTL gave this performance on a cross-subject setting while EEG-NAC and EEG-CSAC both were applied in withinsubject setting. Finally, the comparison with EEGNet also shows better performance in favor of the proposed method as it achieved $+11.5 \%$ more average accuracy with around $10 \%$ less standard deviation. It is to be noted similar to BCI Competition IV-2a, in this dataset also it was a balanced binary classification problem where the chance level accuracy is $50 \%$. The same leave-one-out approach was followed for calculating the cross-subject accuracy as described before.
The comparison of performance of LR-TSTL between healthy subject and patient populations is shown in Fig. 5. Here, we can see that the median accuracy is higher in patients than in healthy participants and variation of performance is also less than the healthy. This is an interesting observation since the inter-subject variability is higher in patients than in healthy due to altered neurodynamics after stroke. This could be a indirect indication that the proposed method has the ability to find more generalizable features from a diverse data distribution and hence can resolve the non-stationary effects in a more effective manner. Additionally, we have analysed the effect of leaving more than one subject out for the cross-subject classification performance. To this end, we have started with one subject and then kept on adding one extra subject into the training dataset until we exhaust the list keeping only the target subject out. Thus we got the accuracy variation for all subjects which was then averaged to get the graph presented in Fig. 4. Here we can see that the average classification accuracy is improved as we have added more subjects in to the training dataset. The error bars in the graph shows the standard error of the mean across all the subjects which is a bit high due to large inter-subject variability in the feature patterns. Additionally, it can be attributed to the fact that sometimes the addition of new data which in spite of being good quality do not represent the test data of the target subject which can be a source of performance degradation.

Thus the proposed LR-TSTL method established its superior performance both in the case of healthy and patient datasets over existing state-of-the-art methods. The ability of LRTSTL to achieve comparable performance between healthy and patient datasets in a cross-subject setting makes it suitable for a versatile practical applications for a wider population. The method shows promising results to realise calibration free BCI systems which can significantly enhance the usability of this technology. It can reduce patients' frustration during a BCI based rehabilitation setting where a patient had to go through the monotonous calibration procedure every time a new therapy session starts. This would give them more time for mental practice and receiving neurofeedback during the same therapy session which is essential of effective motor functional recovery. Hence, the future work will concentrate on conducting pilot trials on stroke patients in a calibra- 


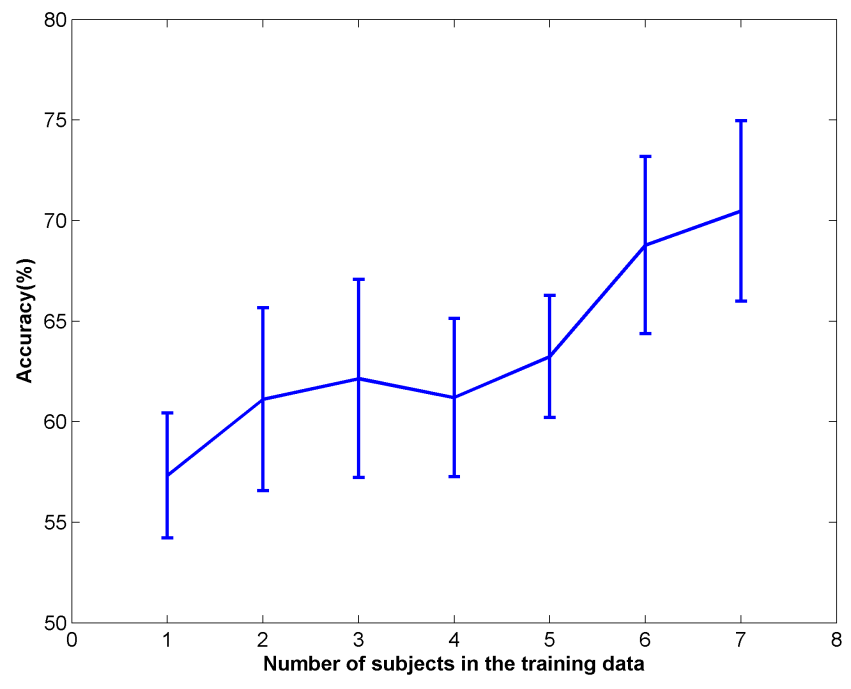

Fig. 4. The effect of leaving more subject out on the cross-subject classification.

tion free BCI enviroment to validate the clinical efficacy of the propsoed LR-TSTL method while providing continuous multimodal neurofeedback. The advantage of the proposed approach over the other approaches is partially attributed to the use of powerful Riemannian concept of tangent space which resolves the confusion in assigning class membership to features in an highly noisy situation. The improvement in results can be further attributed to the fact that tangent space based logistic regression was coupled with SubjectSpecific Multivariate Empirical Mode Decomposition which contributed to the denoising and helped to identify subject specific characteristics in the feature distribution which ultimately led to achieve more generalizable features essential for enhancing the cross-subject accuracy.

One of the limitation of the proposed method is its time complexity. As the method involves the computation of MEMD which has time complexity of $\mathrm{O}(\mathrm{n} \log \mathrm{n})$ [49] where $\mathrm{n}$ is the data length. Additionally, Riemannian Geometry (RG) has complexity $\mathrm{O}(\mathrm{n} 3)(\mathrm{n}=$ number of EEG electrodes). Therefore, the real time implementation of method depends on the use of additional hardware such as dependancy on parallel computing architecture such as Cuda or hardware resources such as FPGA. Hence, the future work should focus on the development of new formulations which can reduce the computational complexity of the method or on the deployment of the method in an suitable computational architecture for realtime implementation.

In recent years several deep learning approaches are proposed for reducing the burden of calibration in BCI design. For example, separated channel convolutional network is proposed on top of CSP as a transfer learning method [50]. A hybrid deep neural network combining Convolutional neural network and Long Short-Term Memory is also proposed for transfer learning which gave satisfactory performance in calibrating the BCI for a new subject with fewer training examples [51]. As the inter-subject variability is a major hindrance in the path of realising transfer learning based BCI Zhang and colleagues proposed 5 new schemes for fine tuning the deep learning BCI approaches [52]. However, the results are still inconclusive to say whether the deep learning based approaches have a clear advantage on the traditional approaches of transfer learning based BCI such as Riemannian Geometry and others unless a direct comparison is made across various datasets, the scope of which is limited in this paper.

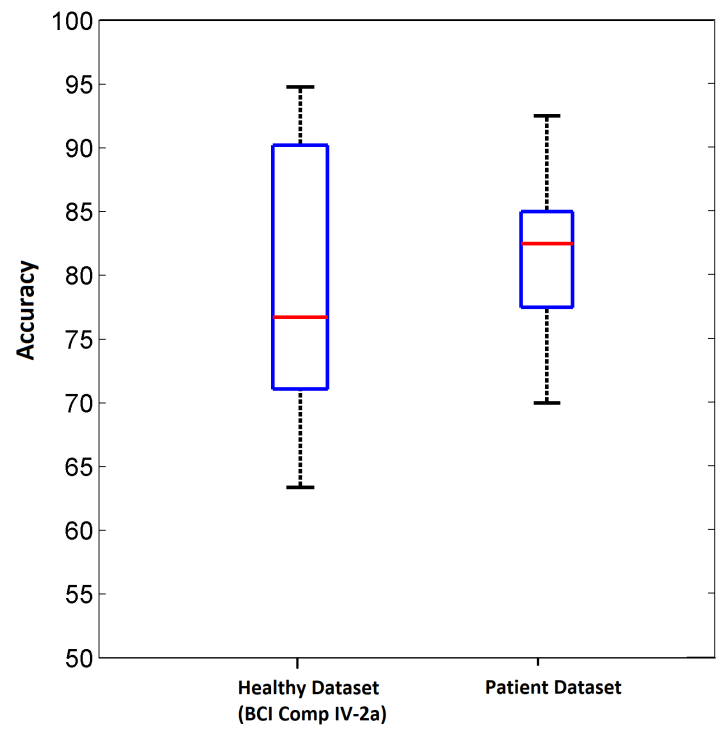

Fig. 5. Comparison of performance between Healthy (BCI Competition IV2a) and Stroke patients dataset for the proposed LR-TSTL method.

\section{CONCLUSION}

This paper presents a fusion of a logistic regression algorithm using tangent space-based transfer learning (LR-TSTL) EEG signal analysis for the classification of binary MI tasks. The features of the MI EEG signals are determined by the tangent space approach in the adopted system, and the derived features are used for classification as inputs to the logistic regression model. The reported method is ideal for representative extraction of the MI data and tangent space logistic regression is an effective classifier to distinguish the characteristics of the MI tasks. For classification of independent variables n(n + 1)/2 features are taken into account by considering the lower triangular matrix of the $\mathrm{n} x \mathrm{n}$ given features. The experimental evaluation is conducted on BCI Competition IV dataset $2 \mathrm{a}$ (for healthy subjects) and on a stroke patients' dataset. The novelty in the proposed approach is to apply subject-specific MEMDbased filtering in the pre-processing step to reduce the effect of intra-subject and inter-subject non-stationarity in the EEG signals. This pre-processing stage provides improved EEG signals from which the distributions of the extracted features show statistically significant differences. The cross-subject learning performance of the proposed LR-TSTL method is compared with three state-of-the-art within-subject learning methods which gave a comparable or even better performance in terms of average classification accuracy in the case of healthy subjects. Moreover, in the case of the patient dataset LR-TSTL has significantly outperformed the current benchmark accuracy giving more stable and robust performance than 
TABLE III

LOW AND HIGH-FREQUENCY VALUES IN HZ WITH CLASSIFICATION ACCURACY (\%) FOR THE PROPOSED METHOD (LR-TSTL) ON LEFT VERSUS RIGHT HAND TASK ON IN-HOUSE RECORDED PATIENT EEG DATA.

\begin{tabular}{cccccccc}
\hline Subject & $\begin{array}{c}\text { Low } \\
\text { Frequency }\end{array}$ & $\begin{array}{c}\text { High } \\
\text { Frequency }\end{array}$ & $\begin{array}{c}\text { Proposed } \\
\text { Method LvR }\end{array}$ & $\begin{array}{c}\text { Kappa } \\
\text { value }\end{array}$ & EEG-NAC & EEG-CSAC & EEGNet \\
\hline P01 & 6 & 24 & 85 & 0.7 & 70 & 72.5 & 67.5 \\
P02 & 6 & 24 & 85 & 0.7 & 67.5 & 72.5 & 92.5 \\
P03 & 8 & 24 & 75 & 0.5 & 75 & 82.5 & 52.5 \\
P04 & 6 & 24 & 82.5 & 0.65 & 65 & 72.5 & 67.5 \\
P05 & 8 & 26 & 77.5 & 0.55 & 75 & 77.5 & 60 \\
P06 & 7 & 27 & 70 & 0.4 & 67.5 & 72.5 & 75 \\
P07 & 6 & 30 & 90 & 0.8 & 67.5 & 75 & 92.5 \\
P08 & 7 & 25 & 92.5 & 0.85 & 72.5 & 75 & 52.5 \\
P09 & 8 & 26 & 82.5 & 0.65 & 72.5 & 82.5 & 52.5 \\
P10 & 6 & 24 & 77.5 & 0.55 & 70 & 75 & 90 \\
\hline Average & & & 81.75 & 0.635 & 70.25 & 75.75 & 70.25 \\
Std & & & 6.88 & 0.13 & 3.42 & 3.91 & 16.56 \\
\hline
\end{tabular}

in healthy subjects as revealed by the box plots of accuracy distribution. Thus the impact of the proposed method on the existing BCI technology is two-fold: firstly, it showed that the performance of the cross-subject learning performance can be enhanced to the within-subject learning level and beyond so that calibration-free BCI systems can be practically feasible; and secondly, it showed more robust and enhanced performance in the stroke patients case whereby the EEG nonstationarity is an even a larger issue than in healthy subjects. Hence, it can be argued that the proposed method has the potential to pave the way for the next generation of calibrationfree $\mathrm{BCI}$ technologies which can be more practically usable especially in the case of neurorehabilitative BCI designs for stroke patients. In the future, evaluating the proposed method with fewer features could be interesting and also expanding the proposed method (LR-TSTL) to assign the EEG or potentially MEG into more than four classes or multi-class classification.

\section{REFERENCES}

[1] J. R. Wolpaw, D. J. McFarland, G. W. Neat, and C. A. Forneris, "An EEG-based brain-computer interface for cursor control," Electroencephalography and clinical neurophysiology, vol. 78, no. 3, pp. 252-259, 1991.

[2] J. Wolpaw and E. W. Wolpaw, Brain-computer interfaces: principles and practice. OUP USA, 2012.

[3] R. T. Toll et al., "An electroencephalography connectomic profile of posttraumatic stress disorder," American Journal of Psychiatry, vol. 177, no. 3, pp. 233-243, 2020. PMID: 31964161.

[4] Y. Zhang, W. Wu, R. T. Toll, et al., "Identification of psychiatric disorder subtypes from functional connectivity patterns in resting-state electroencephalography," Nat Biomed Eng, 2020.

[5] M. C. Meyer, E. S. van Oort, and M. Barth, "Electrophysiological correlation patterns of resting state networks in single subjects: a combined EEG-fMRI study," Brain topography, vol. 26, no. 1, pp. 98109, 2013.

[6] S. Saha and M. Baumert, "Intra- and inter-subject variability in eegbased sensorimotor brain computer interface: A review," Frontiers in Computational Neuroscience, vol. 13, p. 87, 2020.

[7] A. Chowdhury, Y. K. Meena, H. Raza, B. Bhushan, A. K. Uttam, N. Pandey, A. A. Hashmi, A. Bajpai, A. Dutta, and G. Prasad, "Active physical practice followed by mental practice using bci-driven hand exoskeleton: A pilot trial for clinical effectiveness and usability," IEEE Journal of Biomedical and Health Informatics, vol. 22, no. 6, pp. 17861795,2018

[8] P. Gaur, R. B. Pachori, H. Wang, and G. Prasad, "A multi-class EEGbased BCI classification using multivariate empirical mode decomposition based filtering and Riemannian geometry," Expert Systems with Applications, vol. 95, pp. 201-211, 2018.
[9] H. Raza, H. Cecotti, Y. Li, and G. Prasad, "Adaptive learning with covariate shift-detection for motor imagery-based brain-computer interface," Soft Computing, vol. 20, no. 8, pp. 3085-3096, 2016.

[10] F. Lotte and C. Guan, "Regularizing common spatial patterns to improve BCI designs: unified theory and new algorithms," IEEE Transactions on biomedical Engineering, vol. 58, no. 2, pp. 355-362, 2010.

[11] V. Jayaram, M. Alamgir, Y. Altun, B. Scholkopf, and M. GrosseWentrup, "Transfer learning in brain-computer interfaces," IEEE Computational Intelligence Magazine, vol. 11, no. 1, pp. 20-31, 2016.

[12] P. Gaur, K. McCreadie, R. B. Pachori, H. Wang, and G. Prasad, “Tangent Space Features-Based Transfer Learning Classification Model for TwoClass Motor Imagery BrainComputer Interface," International Journal of Neural Systems, vol. 29, no. 10, p. 1950025, 2019. PMID: 31711330.

[13] N. Robinson, A. P. Vinod, K. K. Ang, K. P. Tee, and C. T. Guan, "EEGbased classification of fast and slow hand movements using waveletCSP algorithm," IEEE Transactions on Biomedical Engineering, vol. 60, no. 8, pp. 2123-2132, 2013.

[14] Y. Yang, S. Chevallier, J. Wiart, and I. Bloch, "Time-frequency optimization for discrimination between imagination of right and left hand movements based on two bipolar electroencephalography channels," EURASIP Journal on Advances in Signal Processing, vol. 2014, no. 1, p. 38, 2014.

[15] H. Ramoser, J. Muller-Gerking, and G. Pfurtscheller, "Optimal spatial filtering of single trial EEG during imagined hand movement," IEEE transactions on rehabilitation engineering, vol. 8, no. 4, pp. 441-446, 2000.

[16] B. Blankertz, R. Tomioka, S. Lemm, M. Kawanabe, and K.-R. Muller, "Optimizing spatial filters for robust EEG single-trial analysis," IEEE Signal processing magazine, vol. 25, no. 1, pp. 41-56, 2007.

[17] S. Roy, D. Rathee, A. Chowdhury, K. McCreadie, and G. Prasad, "Assessing impact of channel selection on decoding of motor and cognitive imagery from MEG data," Journal of Neural Engineering, vol. 17, p. 056037, oct 2020.

[18] Blankertz, Benjamin and Muller, K-R and Curio, Gabriel and Vaughan, Theresa $\mathrm{M}$ and Schalk, Gerwin and Wolpaw, Jonathan R and Schlogl, Alois and Neuper, Christa and Pfurtscheller, Gert and Hinterberger, Thilo and others, "The BCI competition 2003: progress and perspectives in detection and discrimination of EEG single trials," IEEE transactions on biomedical engineering, vol. 51, no. 6, pp. 1044-1051, 2004.

[19] B. Blankertz, K.-R. Muller, D. J. Krusienski, G. Schalk, J. R. Wolpaw, A. Schlogl, G. Pfurtscheller, J. R. Millan, M. Schroder, and N. Birbaumer, "The BCI competition III: Validating alternative approaches to actual BCI problems," IEEE transactions on neural systems and rehabilitation engineering, vol. 14, no. 2, pp. 153-159, 2006.

[20] B. Blankertz, R. Tomioka, S. Lemm, M. Kawanabe, and K.-R. Muller, "Optimizing spatial filters for robust EEG single-trial analysis," Signal Processing Magazine, IEEE, vol. 25, no. 1, pp. 41-56, 2008.

[21] P. Gaur, H. Gupta, A. Chowdhury, K. McCreadie, R. B. Pachori, and H. Wang, "A sliding window common spatial pattern for enhancing motor imagery classification in eeg-bci," IEEE Transactions on Instrumentation and Measurement, vol. 70, pp. 1-9, 2021.

[22] P. Gaur, K. McCreadie, R. B. Pachori, H. Wang, and G. Prasad, "An automatic subject specific channel selection method for enhancing motor imagery classification in eeg-bci using correlation," Biomedical Signal Processing and Control, vol. 68, p. 102574, 2021. 
[23] Y. Zhang, C. S. Nam, G. Zhou, J. Jin, X. Wang, and A. Cichocki, "Temporally constrained sparse group spatial patterns for motor imagery bci," IEEE Transactions on Cybernetics, vol. 49, no. 9, pp. 3322-3332, 2019.

[24] Y. Jiao, Y. Zhang, X. Chen, E. Yin, J. Jin, X. Wang, and A. Cichocki, "Sparse group representation model for motor imagery eeg classification," IEEE Journal of Biomedical and Health Informatics, vol. 23, no. 2, pp. 631-641, 2019.

[25] B. Blankertz, G. Dornhege, M. Krauledat, K.-R. Müller, and G. Curio, "The non-invasive Berlin brain-computer interface: fast acquisition of effective performance in untrained subjects," NeuroImage, vol. 37, no. 2 , pp. 539-550, 2007.

[26] K. K. Ang, Z. Y. Chin, C. Wang, C. Guan, and H. Zhang, "Filter bank common spatial pattern algorithm on BCI competition IV datasets $2 \mathrm{a}$ and 2b," Frontiers in Neuroscience, vol. 6, 2012.

[27] F. Lotte and C. Guan, "Spatially regularized common spatial patterns for EEG classification," in 2010 20th International Conference on Pattern Recognition, pp. 3712-3715, IEEE, 2010.

[28] H. Lu, K. N. Plataniotis, and A. N. Venetsanopoulos, "Regularized common spatial patterns with generic learning for eeg signal classification," in Engineering in Medicine and Biology Society, 2009. EMBC 2009. Annual International Conference of the IEEE, pp. 6599-6602, IEEE, 2009.

[29] B. Blankertz, M. Kawanabe, R. Tomioka, F. Hohlefeld, K.-r. Müller, and V. V. Nikulin, "Invariant common spatial patterns: Alleviating nonstationarities in brain-computer interfacing," in Advances in neural information processing systems, pp. 113-120, 2008

[30] H. Kang, Y. Nam, and S. Choi, "Composite common spatial pattern for subject-to-subject transfer," IEEE Signal Processing Letters, vol. 16, no. 8, pp. 683-686, 2009.

[31] P. Gaur, R. B. Pachori, H. Wang, and G. Prasad, "An empirical mode decomposition based filtering method for classification of motorimagery EEG signals for enhancing brain-computer interface," in Neural Networks (IJCNN), 2015 International Joint Conference on, pp. 1-7, IEEE, 2015.

[32] P. Gaur, R. B. Pachori, H. Wang, and G. Prasad, "A multivariate empirical mode decomposition based filtering for subject independent BCI," in 2016 27th Irish Signals and Systems Conference (ISSC), pp. 17, IEEE, 2016.

[33] P. Gaur, R. B. Pachori, H. Wang, and G. Prasad, "An Automatic Subject Specific Intrinsic Mode Function Selection for Enhancing Two-Class EEG based Motor Imagery-Brain Computer Interface,” IEEE Sensors Journal, 042019.

[34] N. E. Huang, Z. Shen, S. R. Long, M. C. Wu, H. H. Shih, Q. Zheng, N.C. Yen, C. C. Tung, and H. H. Liu, "The empirical mode decomposition and the Hilbert spectrum for nonlinear and non-stationary time series analysis," in Proceedings of the Royal Society of London A: Mathematical, Physical and Engineering Sciences, vol. 454, pp. 903-995, The Royal Society, 1998.

[35] Park, Cheolsoo and Looney, David and ur Rehman, Naveed and Ahrabian, Alireza and Mandic, Danilo P, "Classification of motor imagery BCI using multivariate empirical mode decomposition," IEEE Transactions on neural systems and rehabilitation engineering, vol. 21, no. 1 , pp. 10-22, 2012.

[36] F. Lotte and C. Guan, "Regularizing common spatial patterns to improve BCI designs: unified theory and new algorithms," IEEE Transactions on biomedical Engineering, vol. 58, no. 2, pp. 355-362, 2011.

[37] A. Chowdhury, H. Raza, Y. K. Meena, A. Dutta, and G. Prasad, "Online covariate shift detection-based adaptive braincomputer interface to trigger hand exoskeleton feedback for neuro-rehabilitation," IEEE Transactions on Cognitive and Developmental Systems, vol. 10, no. 4 pp. 1070-1080, 2018

[38] Y. Yang, S. Chevallier, J. Wiart, and I. Bloch, "Subject-specific timefrequency selection for multi-class motor imagery-based bcis using few laplacian eeg channels," Biomedical Signal Processing and Control, vol. 38, pp. 302-311, 2017.

[39] A. K. Das, S. Suresh, N. Sundararajan, and K. Subramanian, "A subject-specific frequency band selection for efficient bci- an interval type-2 fuzzy inference system approach," in 2015 IEEE International Conference on Fuzzy Systems (FUZZ-IEEE), pp. 1-8, 2015.

[40] H.-I. Suk and S.-W. Lee, "Subject and class specific frequency bands selection for multiclass motor imagery classification," International Journal of Imaging Systems and Technology, vol. 21, no. 2, pp. 123-130, 2011.

[41] Park, Cheolsoo and Looney, David and Ahrabian, Alireza and Mandic, Danilo P and others, "Classification of motor imagery BCI using mul- tivariate empirical mode decomposition," IEEE Transactions on Neural Systems and Rehabilitation Engineering, vol. 21, no. 1, pp. 10-22, 2013.

[42] R. B. Pachori, "Discrimination between ictal and seizure-free EEG signals using empirical mode decomposition," Research Letters in Signal Processing, vol. 2008, p. 14, 2008

[43] A. Barachant, S. Bonnet, M. Congedo, and C. Jutten, "Multiclass brain-computer interface classification by Riemannian geometry," IEEE Transactions on Biomedical Engineering, vol. 59, no. 4, pp. 920-928, 2012.

[44] A. Barachant, S. Bonnet, M. Congedo, and C. Jutten, "Riemannian geometry applied to bci classification," in Latent Variable Analysis and Signal Separation (V. Vigneron, V. Zarzoso, E. Moreau, R. Gribonval, and E. Vincent, eds.), (Berlin, Heidelberg), pp. 629-636, Springer Berlin Heidelberg, 2010.

[45] W. Förstner and B. Moonen, A Metric for Covariance Matrices, pp. 299309. Berlin, Heidelberg: Springer Berlin Heidelberg, 2003.

[46] Hosmer Jr, David W and Lemeshow, Stanley and Sturdivant, Rodney X, Applied logistic regression, vol. 398. John Wiley \& Sons, 2013.

[47] V. J. Lawhern, A. J. Solon, N. R. Waytowich, S. M. Gordon, C. P. Hung, and B. J. Lance, "EEGNet: a compact convolutional neural network for EEG-based brain-computer interfaces," Journal of Neural Engineering, vol. 15 , p. 056013 , jul 2018

[48] H. Raza, A. Chowdhury, and S. Bhattacharyya, "Deep learning based prediction of eeg motor imagery of stroke patients for neurorehabilitation application," in 2020 International Joint Conference on Neural Networks (IJCNN), pp. 1-8, 2020.

[49] L.-C. Wu, H.-H. Chen, J.-T. Horng, C. Lin, N. E. Huang, Y.-C. Cheng, and K.-F. Cheng, "A novel preprocessing method using hilbert huang transform for maldi-tof and seldi-tof mass spectrometry data," PLOS ONE, vol. 5, pp. 1-15, 082010

[50] X. Zhu, P. Li, C. Li, D. Yao, R. Zhang, and P. Xu, "Separated channel convolutional neural network to realize the training free motor imagery bci systems," Biomedical Signal Processing and Control, vol. 49, pp. 396-403, 2019.

[51] R. Zhang, Q. Zong, L. Dou, X. Zhao, Y. Tang, and Z. Li, "Hybrid deep neural network using transfer learning for eeg motor imagery decoding," Biomedical Signal Processing and Control, vol. 63, p. 102144, 2021.

[52] K. Zhang, N. Robinson, S.-W. Lee, and C. Guan, "Adaptive transfer learning for eeg motor imagery classification with deep convolutional neural network," Neural Networks, vol. 136, pp. 1-10, 2021. 